\title{
BNNG Algorithm Modeling for Vehicle Classification Recognition under Non Line-of -sight Environment
}

\author{
WU Long ${ }^{1,2, *}$, Huang Kaifeng ${ }^{1,2}$, and Feng Juqiang ${ }^{1,2}$ \\ ${ }^{1}$ School of Mechanical and Electrical Engineering Huainan Normal University, 232038,Huainan, China \\ ${ }^{2}$ Research Center for Power Supply and Control Engineering Technology of Rail Transit, 232038, Huainan, China.
}

\begin{abstract}
At present, the automatic classification of vehicles on roads is mostly based on image recognition, and there are defects in adaptability under non-line-of-sight environments. In this paper, based on the similarity of the integration of the ecosystem model and multi-neural network model, an artificial neural network group (BNNG) algorithm was proposed. The vehicle's driving acoustic signal was taken as the research object, and it was calculated using the Artificial Neural Network (BNNG) algorithm to achieve automatic classification and recognition of vehicle models. Through experimental tests, it is shown that under non-line-of-sight environments, the accuracy of vehicle classification can be improved, and the misrecognition rate of similar models can be greatly reduced. This provided a new method for the automatic classification and identification of vehicles on roads, which was of great significance to monitor vehicle safety in non-line-of-sight environments.
\end{abstract}

\section{Introduction}

At present, the classification of vehicles is mainly within the line-of-sight range. Vehicles are distinguished based on the degree of road damage and occupancy in the process of driving, which is the basis for traffic statistics, road toll determination, and vehicle restrictions. Most of the traditional vehicle classification methods are based on image recognition. However, there are problems such as environmental adaptability and lack of versatility when used in practice. This article takes the acoustic signals in the vehicle driving process as research objects and uses the Artificial Neural Network (BNNG) algorithm to realize automatic classification of vehicles under non-line-of-sight environments[1].

Neural network ensemble is the use of a finite number of neural networks to learn the same problem, and the output is determined by each neural network output in accordance with certain rules. The research on neural network integration mainly focuses on two aspects: how to generate an integrated network and how to determine the output rules. Domestic and foreign scholars have proposed Boosting, Bagging, ALA, OLA, PLA $[2,3]$ and other algorithms which have studied this aspect, but the problem of black box inside the neural network still exists. It is urgent to find a model to explore the internal learning mechanism of network integration. After previous experiments, this paper finds that there are certain similarities between the neural network integration and the evolution model of the ecosystem. For this purpose, the ecosystem model is predicted and matched with the parameters in the neural network integration. Using evolutionary rules to guide the integration of neural networks, we propose an artificial neural network group.

\section{Artificial neural network group (BNNG) algorithm design}

\subsection{The BNNG model proposed}

Ecosystem refers to the biological community and its living environment. Through the exchange of materials, energy conversion and information transmission, biological communities occupies a certain space, has a certain structure, performs certain functions, and become a dynamic equilibrium. It is a perfect dynamic in nature. Its internal evolution based on mutual assistance, competition and other rules to complete. Multi-neural networks work in a population-based manner and evolve according to the rules of ecosystem evolution. Therefore, they are called artificial neural network groups. This paper does a predictive match for the two models. Table 1 only lists some of the matching rules:

According to the basic rules in Table 1, this article proposes the following criteria: (1) If the environment variables $X^{M}$ are limited, the species $f_{i}$ must be limited, that is, the value of $N$ is finite, and the best value $N$ can be obtained based on the law of conservation of energy and distribution; (2) If environmental variables $X^{M}$ different, the species needed are different, that is, the

\footnotetext{
* Corresponding author: Wu long: fjq5060912@126.com
} 
Table 1. BNNG model matching.

\begin{tabular}{cccccccc}
\hline model & Match 1 & Match 2 & Match 3 & Match 4 & Match 5 & Match 6 & Match 7 \\
\hline ecosystem & $\begin{array}{c}\text { environme } \\
\text { ntal factor }\end{array}$ & Species & $\begin{array}{c}\text { Population } \\
\text { relations }\end{array}$ & $\begin{array}{c}\text { Evolutionary } \\
\text { rules }\end{array}$ & $\begin{array}{c}\text { Interspeci } \\
\text { es } \\
\text { difference }\end{array}$ & $\begin{array}{c}\text { Survival } \\
\text { ratio }\end{array}$ & $\begin{array}{c}\text { Ecological } \\
\text { balance }\end{array}$ \\
\hline $\begin{array}{c}\text { Neural } \\
\text { network } \\
\text { group }\end{array}$ & $\begin{array}{c}\text { Input } \\
\text { variable }\end{array}$ & $\begin{array}{c}\text { Neural } \\
\text { Networks }\end{array}$ & $\begin{array}{c}\text { Network } \\
\text { structure }\end{array}$ & $\begin{array}{c}\text { Type weight } \\
\text { change }\end{array}$ & $\begin{array}{c}\text { Degree of } \\
\text { difference }\end{array}$ & $\begin{array}{c}\text { Impact } \\
\text { factor }\end{array}$ & $\begin{array}{c}\text { Expected } \\
\text { output }\end{array}$ \\
\hline $\begin{array}{c}B N N G \\
X^{M}\end{array}$ & $f_{i}$ & $\omega_{i}$ & $\begin{array}{c}\omega_{i} \\
\text { Adjustment }\end{array}$ & $A_{i}$ & $\Delta_{i}$ & $T^{n}$ \\
\hline $\begin{array}{c}\text { distribution is different and the types N needed are } \\
\text { naje }\end{array}$ & & $W E=\int p(x)[\bar{f}(x)-t(x)]^{2} d x$
\end{tabular}

different. (3) There are major species and minor species in the ecosystem, so the neural network groups are divided into the main network and the incentive network, and the selection process follows the principle of similarity and difference respectively. (4) The increase of species $f_{i}$ will produce impact factors $\Delta$, which will be fed back into each species and eventually make the system one of the four states of balance/damage/rebalance/destruction.

\subsection{BNNG algorithm process}

It is assumed that the learning objective of the neural network group is to simulate the evolution function: $f: X^{m} \rightarrow T^{n}$. The neural network group is composed of $N$ neural networks (populations) $f_{1}, f_{2}, f_{3}, \bullet \bullet \cdot f_{N}$. Each network is assigned a weight $\omega_{i}$, satisfying the conditions $\omega_{i} \geq 0$, and $\sum_{i=1}^{N} \omega_{i}=1$. The weight adjustment rule is determined by the influence factor $\Delta$, and the specific definition is later defined. The output of the neural network group is generated by the weighted average of the participating neural networks. The output value in the group is determined by equation (1), where $f_{i l}$ is the output value of the member network within the group.

$$
\bar{f}_{l}=\sum_{i=1}^{N} \omega_{i} f_{i l}
$$

Set up $x \in X^{m}$ to meet the distribution of $p(x)$. If the output $t(x)$ is in the input destination $x$, the output of $f_{i}$ the first member network is $f_{i}(x)$, then the neural network group outputs under the input $x$ is .

$$
\bar{f}(x)=\sum_{i=1}^{N} \omega_{i} f_{i}(x)
$$

Define the following quantities: In addition to the $i$ member network $f_{i}$ in the neural network group, the rest of the member networks are integrated in the output of the input $x$.

$$
\bar{f}_{-i}(x)=\sum_{j=1, j \neq i}^{N} \frac{\omega_{j}}{1-\omega_{i}} f_{j}(x)
$$

Global generalization error of neural network groups:
The mero generalization error of the $i$ member network $f_{i}$ of the neural network group:

$$
M E_{i}=\int p(x)[\bar{f}(x)-t(x)]^{2} d x
$$

The degree of difference between member networks $f_{i}$ and neural network groups $\bar{f}$ :

$$
A_{i}=\int p(x)\left[f_{i}(x)-\bar{f}(x)\right]^{2} d x
$$

The degree of difference between member networks $f_{i}$ and neural network groups $\bar{f}_{-i}$ :

$$
A_{-i}=\int p(x)\left[f_{i}(x)-\bar{f}_{-i}(x)\right]^{2} d x
$$

The weighted average of the globalization error of the neural network group:

$$
\overline{W E}=\sum_{i=1}^{N} \omega_{i} M E_{i}
$$

A weighted average of the degree of difference between member networks and neural network groups:

$$
\bar{A}=\sum_{i=1}^{N} \omega_{i} A_{i}
$$

Through the integrated theoretical analysis of Krogh and Vedelsby in 1995, it can be concluded that [4-7]:

$$
W E=\overline{W E}-\bar{A}
$$

Formula (3), (6), (7) can be drawn.

$$
A_{i}=\left(1-\omega_{i}\right)^{2} A_{-i}
$$

Suppose $W E * A_{i}=\omega_{i}\left(M E_{i}-A_{i}\right)$, we can get from equations (8), (9), (10), (11).

$$
\sum_{i=1}^{N} W E * A_{i}=\sum_{i=1}^{N} \omega_{i}\left[M E_{i}-\left(1-\omega_{i}\right)^{2} A_{-i}\right]=W E
$$

It can be seen that the smaller the local generalization error $M E_{i}$ is, the greater the degree of difference $A_{-i}$ between the network $f_{i}$ and the neural network group $\bar{f}_{-i}$ is, and the smaller the global generalization error $W E$ is. Therefore, a global generalization error threshold $\mathrm{T}$ needs to be set.

Set up a new incentive network: $f_{K}(x), K \in Z$. In the principle of biological evolution, the best type $f_{K}(x)$ is the basic $\mathrm{NN}$ network, and it is even more difficult to break the balance of the original system. 
Every time it is introduced, it will have an impact factor $\Delta$ on the weight, which is defined as:

$$
\Delta_{i}=\frac{1}{A_{-i} * M E_{i}}
$$

Impact factor normalization.

$$
\bar{\Delta}=\frac{\Delta}{\Delta_{\max }}
$$

The purpose is to prevent training from falling into local minimum values and to increase the stability of the neural network group. If the network is similar, then $\bar{\Delta}$ are all equal. The output of the neural network group is similar to the average output of the neural network integration. If there are differences in the network, the evolution of impact factors will be used to promote the development of the new system to a new balance. The weights of each member network of the whole neural network group are as follows: $\omega_{i}=\bar{\Delta} * \omega_{i-1}$. To meet the conditions $\omega_{i} \geq 0$.

$$
\sum_{i=1}^{N} \omega_{i}=1
$$

The system body network and the incentive network are trained repeatedly according to the above steps. When $W E$ is reduced to $\mathrm{T}$, the training ends and the new balance is output. The neural network group algorithm completes the convergence.

\section{Experimental verification of BNNG algorithm}

\subsection{Signal Acquisition}

This experiment was conducted at night and in foggy days (non-line-of-sight environment) at the 206 National Road in Huainan for more than ten consecutive days to collect acoustic signals from passing vehicle. The data acquisition card used four-channel data acquisition card from Beijing Kerui Company. And acoustic signal sensors used the condenser microphones and matching preamplifiers of Hangzhou Aihua Company. The HV300 laser speedometer was used to measure the vehicle speed.

In order to facilitate research and improve the accuracy of data, we select the vehicle speed in the range of $40 \mathrm{~km} / \mathrm{h}-80 \mathrm{~km} / \mathrm{h}$, and the speed distribution is the same as the sample. Finally, large passenger vehicles
(Jinlong CCJ6100, abbreviated as CC), medium-sized passenger buses (Yutong MD6825, abbreviated as MD), small cars (Chery A5, abbreviated as A), trucks (liberation J142, abbreviated as J) were selected. Each of the four vehicle types has 40 samples, and divided into 2 groups, one group for training samples and one group for testing samples. Expected output $T(x)=[00,1,10,11]$.

\subsection{Determining AR Model Order}

There are many methods for determining the AR model order. This article determines the final prediction error (FPE) criterion based on multiple experiments.

$$
\operatorname{FPE}(k)=\sigma_{k}^{2}\left(\frac{N+k+1)}{N \quad k+1}\right.
$$

Among them $\mathrm{k}$ is the model order and $\sigma_{k}^{2}$ is the variance of the excitation source of the $\mathrm{k}$-th order model, and $\mathrm{N}$ is the number of sample data points. As the $\mathrm{k}$ increases, the inaccuracy of the prediction error estimation increases, and the numerical value $\sigma_{k}^{2}$ decreases, so with the number increases, FPE will have a minimum value, and the corresponding order of the minimum value is the last determined order. By calculation, the order corresponding to the minimum value of FPE in this paper is 8[8-9].

\subsection{Signal Acquisition}

According to the $\mathrm{u}$ check value from small to large, the redundant parameters were deleted one by one to constitute a different combination of parameters. As a BNNG input vector, 1 is output when output $\geqslant 0.5$, and 0 is output when output $<0.5$. This time selected 40 training samples and 40 test samples.

$$
\begin{gathered}
u=\frac{\overline{X_{1}}-\overline{\mathrm{X}_{2}}}{\sqrt{\frac{\mathrm{s}_{1}^{2}}{\mathrm{n}_{1}}+\frac{s_{2}^{2}}{n_{2}}}} \\
H_{0}: \mu_{1}=\mu_{2} ; H_{1}: \mu_{1} \neq \mu_{2}
\end{gathered}
$$

Table 2. Sample input training data section.

\begin{tabular}{|c|c|c|c|c|c|c|c|}
\hline$C C$ & $C C$ & $M D$ & $M D$ & $A$ & $A$ & $J$ & $J$ \\
\hline 1 & 1 & 1 & 1 & 1 & 1 & 1 & 1 \\
\hline-2.2662 & -2.1906 & -3.1468 & -3.1878 & -2.5419 & -2.6952 & -1.8249 & -1.8979 \\
\hline 2.3479 & 2.0332 & 4.2635 & 4.5152 & 2.2286 & 2.6699 & 0.22601 & 0.32907 \\
\hline-2.094 & -1.6246 & -3.149 & -3.6852 & -0.37192 & -0.78451 & 0.67266 & 0.70654 \\
\hline 1.6637 & 1.2135 & 1.3727 & 2.0307 & -0.34879 & -0.35569 & 0.47977 & 0.44619 \\
\hline-1.0867 & -0.72649 & -0.56508 & -1.0761 & -0.42777 & -0.13064 & -0.47657 & -0.5362 \\
\hline
\end{tabular}




\begin{tabular}{|c|c|c|c|c|c|c|c|}
\hline 0.62375 & 0.45733 & 0.36962 & 0.60269 & 0.78063 & 0.57171 & -0.28681 & -0.27251 \\
\hline-0.18669 & -0.1609 & -0.14385 & -0.19833 & -0.30428 & -0.25719 & 0.20991 & 0.22486 \\
\hline
\end{tabular}

\subsection{Determining the BNNG Classifier Body and Incentive Network}

According to the distribution of the input signal and the principle of similarity defined in the algorithm, this article determines the main network as bp, ebp, tbp, cbp four kinds of networks. According to the number of the main network, structure and the principles of the differences specified in the algorithm, we determine that the incentive network are Perceptron, Radial basis (exact fit), Radial basis (fewer neurons), Generalized regression, Linear layer (design).

\subsection{Experimental Results and Analysis}

This article programmed to implement $\mathrm{BNNG}$ algorithm in matlab7.0 environment, a total of 24 kinds of network types were introduced. After many simulations straining, the specific vehicle recognition rate results are shown in Table 3, and the training error curve is shown in Figure 1, 2.

Table 3. Vehicle recognition rate results.

\begin{tabular}{|c|c|c|c|c|c|c|c|c|}
\hline \multirow{2}{*}{$\begin{array}{c}\text { Netwo } \\
r k \\
\text { numb } \\
\text { er }\end{array}$} & \multirow{2}{*}{$\begin{array}{c}\text { Network } \\
\text { name }\end{array}$} & \multirow{2}{*}{ Instructions } & \multirow{2}{*}{ Hidden layer nodes } & \multirow{2}{*}{$\begin{array}{l}\text { Netwo } \\
\text { rk type }\end{array}$} & \multicolumn{4}{|c|}{$\begin{array}{c}\text { Correct recognition rate } \\
(\%)\end{array}$} \\
\hline & & & & & $C C$ & $\begin{array}{l}M \\
D\end{array}$ & $A$ & $J$ \\
\hline 1 & Bp1-3 & $\begin{array}{c}\text { Error back propagation } \\
\text { network }\end{array}$ & $3,5,8$ & 3 & 100 & 75 & $\begin{array}{c}10 \\
0 \\
\end{array}$ & 70 \\
\hline 2 & Cbp1-4 & $\begin{array}{c}\text { Cascaded Back } \\
\text { Propagation Network }\end{array}$ & $3,5,8,10$ & 4 & 100 & 95 & $\begin{array}{c}10 \\
0\end{array}$ & $\begin{array}{c}10 \\
0\end{array}$ \\
\hline 3 & Ebp1-4 & Elman neural network & $2,3,4,5$ & 5 & 100 & 75 & $\begin{array}{c}10 \\
0\end{array}$ & 75 \\
\hline 4 & Tbp1-7 & $\begin{array}{c}\text { Time delay back } \\
\text { propagation network }\end{array}$ & $2,3,4,6,7,10,14$ & 7 & 100 & 60 & $\begin{array}{c}10 \\
0\end{array}$ & 75 \\
\hline 5 & $\begin{array}{c}\text { Main } \\
\text { network }\end{array}$ & $\begin{array}{c}\text { Four main body } \\
\text { network convergence }\end{array}$ & & 19 & 100 & 80 & $\begin{array}{c}10 \\
0\end{array}$ & 75 \\
\hline 6 & $\begin{array}{c}\text { Incentive } \\
\text { network }\end{array}$ & GR, etc. & & 5 & 95 & 75 & $\begin{array}{c}10 \\
0\end{array}$ & 90 \\
\hline 7 & $\begin{array}{l}\text { Overall } \\
\text { network }\end{array}$ & $\begin{array}{l}\text { After introducing the } \\
\text { incentive network }\end{array}$ & & & 100 & 95 & $\begin{array}{c}10 \\
0 \\
\end{array}$ & $\begin{array}{c}10 \\
0\end{array}$ \\
\hline
\end{tabular}

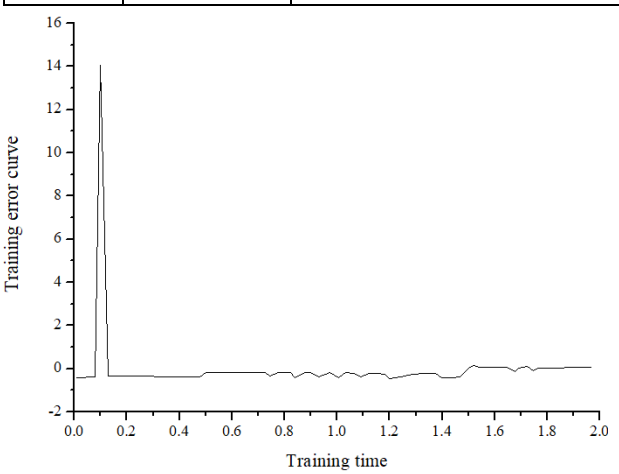

Fig. 1. Single Network Training Error Curve.

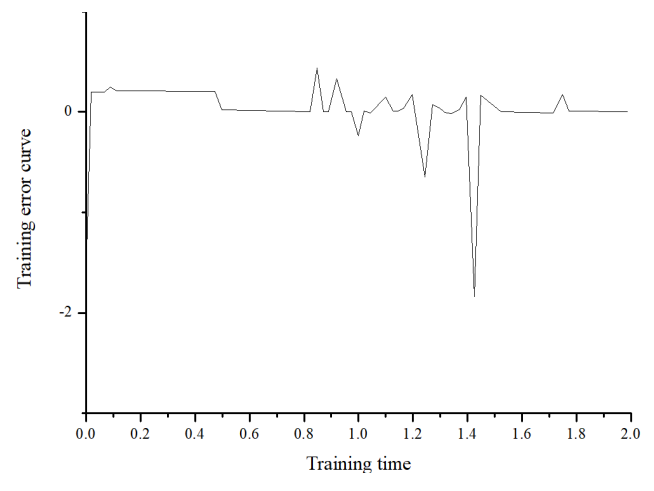

Fig. 2. Neural network group training error curve.
According to the data in Table 3, large passenger cars (CCs) and small cars (A) have obvious characteristic acoustic signals and the lowest misidentification rate of models, while the medium-sized passenger cars (MD) and freight cars $(\mathrm{J})$ have the highest. However, as a whole, it is shown that acoustic signals of different models are used as research objects, and neural network group algorithms can identify vehicle types. Especially under non-line-of-sight environments, the recognition rate of approximate vehicle models can be improved, the generalization error can be reduced, and the overall training time can be increased. The classification recognition improves the verification method. From Fig. 1 and 2, we can see that: (1) There is ambiguity in the constraint relationship between the number of the main network and the input space; (2)The turbulence is caused by the error caused by the incentive network. It is necessary to study the stationary algorithm to improve the robustness.

\section{Conclusion}

In the non-line-of sight environment, taking the acoustic signal of vehicle driving as the research object, and using the bionic neural network group (BNNG) algorithm proposed in this paper, the experimental test shows that the vehicle classification and recognition can be achieved. The accuracy of vehicle classification is 
improved, and the misidentification rate of similar models is greatly reduced. This provides a new method for the automatic classification and identification of vehicles on roads and is of great significance for the monitoring of vehicle safety under non-line-of-sight environments. At the same time, the BNNG algorithm is a new neural network integration model, which establishes and evolves the network according to the rules of ecological evolution. The analysis of experimental data shows that there is a certain similarity between the simulated ecological neural network group and the natural ecological balance model, which provides a theoretical and experimental reference value for the unified framework of neural network integration theory, and also provides a new method and basis for pattern recognition under complex conditions.

\section{Acknowledgment}

The authors would like to thank Research Centre for the Power supply and Control Engineering Technology of Rail Transit for BNNG Algorithm Modeling for Vehicle Classification Recognition under Non Line-of -sight Environment with support (Science Key Fund of Education Department of Anhui province (KJ2017A458), Science Key Fund of huainan normal university (2016xj02zd) And Science Fund of huainan normal university (2018xj32)).

\section{References}

1. Wang Shuangwei, Chen Qiang. Research on Vehicle Sound and Vibration Signal Characteristics of Different Vehicle Models. Acoustics Technology, 3, (2007)

2. Li Kai. A selective neural network integration method based on clustering technology. Computer Research and Development, 42, 4 (2005),

3. Wang Zhengqun. Parallel Learning Neural Network Integration Method. Chinese Journal of Computers, 28, (2005)

4. $\mathrm{Xu}$ Min. Expert system model based on neural network integration $[\mathrm{J}]$. Computer Engineering and Design, 27, 7 (2006)

5. Song Rui, Zhang Jing. An adaptive classification method based on BP neural network group and its application. Chinese Journal of Electronics, 12A (2001)

6. Zhou Zhihua. Neural network integration. Chinese Journal of Computers, January 25 (2005)

7. Wen Xin. MATLAB neural network simulation and application. (Science Press, Beijing, 2003 )

8. Yu Jia, Li Yihua. Research on Automatic Classification of Vehicles Based on Data Mining. Modern Electronic Technology, 9 (2012)

9. Chen Qiang, Li Jiang, Wang Shuangwei. Automatic Vehicle Classification Technology Based on AR Model. Journal of Jilin University (Engineering Science), 2 (2007)
10. Yang Shuling. Carrier frequency measurement based on AR model. Radio Engineering, 36, 9 (2006) 